\title{
Advances In Research Of Spreading Through Air Spaces And The Effects On The Prognosis Of Lung Cancer
}

This article was published in the following Dove Press journal: Cancer Management and Research

\author{
Dandan Cao $\mathbb{D}^{1,2}$ \\ Jun Sha ${ }^{1,2}$ \\ Rui Cui ${ }^{1,2}$ \\ Shuhua Han' \\ 'Department of Pulmonary Medicine, \\ Zhongda Hospital, School of Medicine, \\ South-East University, Nanjing, Jiangsu, \\ People's Republic of China; ${ }^{2}$ Medicine \\ Department of Southeast University, \\ Nanjing, Jiangsu, People's Republic of \\ China
}

\begin{abstract}
The concept of spread through air spaces (STAS) has been described as a new form of invasion in the lung in the 2015 WHO classification of Lung Tumors, namely invasion through alveolar spaces. STAS is a prognostic factor independent of growth pattern and tumor stage, and it is also an independent risk factor for unfavorable prognosis of stage I lung adenocarcinoma (ADC) and stage I lung squamous cell carcinoma (SCC). The pathological characteristics are different between ADC and SCC. STAS is not reported as routine, so setting a unified pathological reading standard, and hunting for STAS as a regular reading process is urgently advocated. We write this review to investigate the research progress of STAS and its effects on the prognosis of lung cancer.
\end{abstract}

Keywords: lung cancer, STAS, pathological characteristics, prognosis

\section{Introduction}

Lung cancer has become the most common incident cancer and the leading cause of cancer-related death. ${ }^{1,2}$ Commonly, tumor spread is the most important factor affecting outcome in malignant tumors. Due to the unique anatomy of the lung, the air spaces are surrounded by the highly regular vascular network of alveoli, and besides being potential routes for lung tumor dissemination, tumor spread through air spaces (STAS) might occur apart from vascular, nodal, and distant spread of tumor cells. STAS $^{3}$ consists of micropapillary clusters, solid nests, or single cells beyond the edge of the tumor into air spaces in the surrounding lung parenchyma, often occurring in early lung adenocarcinoma (ADC). Eight hundred and forty-eight patients with pathological stage I disease who underwent surgery were analyzed by Shiono et al., who found that patients with STAS tend to develop locoregional recurrence and pulmonary metastases. ${ }^{4}$ Warth et al. ${ }^{5}$ demonstrated that a peripheral cell detachment and subsequent intra-alveolar spread of tumor cells is evident at the circumferential tumor edge in many lung ADCs, and micropapillary-predominant ADCs have the highest rates of STAS and the worst related overall and disease-free survival. In addition, STAS itself also faces some problems, such as its existence and detection.

\section{The Mechanism Of STAS}

The mechanism of STAS has not been well studied. It's well known that lung tumor cells could survive after starvation for more than $12 \mathrm{~h}$ during cell culture experiments. We know that some alveolar macrophages live in the air spaces, and the fluid of the
Department of Pulmonary Medicine

Zhongda Hospital, Ding Jia Qiao No. 87,

Nanjing, Jiangsu 210009, People's Republic of China

Email hanshuhua0922@I26.com 
alveolar walls and exudation around the tumor might serve as potential energy sources after cell detachment from the main tumor mass. After the tumor cell gets away from the main tumor lesion, it can be explained by Liotta's ${ }^{6}$ three-step model of invasion: adhere, degrade, and move, but there may be other possible pathways (see Figure 1). Adhesion molecule expression, matrix metalloproteinases (MMPs), laminin, looseness between cancer cells and ultrastructure and stimulation of tumor-infiltrating leukocytes may all contribute to STAS. ${ }^{7}$ Several studies demonstrated that the biological behavior of cancer cells could be influenced by the tumor microenvironment, which is composed of not only cancer cells, but also several kinds of stromal cells. Stromal cells play prominent roles in tumor initiation, progression, and metastasis by secreting soluble factors, such as growth factors or inflammatory chemokines. Wislez et al. ${ }^{8}$ showed that neutrophils can induce tumor shedding and the aerogenous spread of lung ADC with bronchioalveolar carcinoma features, which is related to shorter survival and might be an important event in adenocarcinoma progression. Cancerassociated fibroblasts (CAFs) and tumor-associated macrophages (TAMs) are the major cellular components of the tumor microenvironment which can orchestrate cancer dissemination and metastasis. Qiu et al. ${ }^{9}$ analyzed alpha smooth muscle actin ( $\alpha$-SMA)-positive CAFs and CD204-positive TAMs in 208 resected specimens of stage I-IIIA lung adenocarcinoma. A logistic regression indicated that the presence of STAS is significantly associated with a higher frequency of $\alpha$-SMA-positive CAFs and a higher number of CD204-positive TAMs. The precise molecular mechanisms for STAS may be analyzed using animal models.

\section{STAS And Similar Phenomena}

The 2015 WHO classification of Lung Tumors described STAS as a new form of invasion in lung cancer, which is said to be validated by two large cohorts studies. ${ }^{5,10}$ Arne

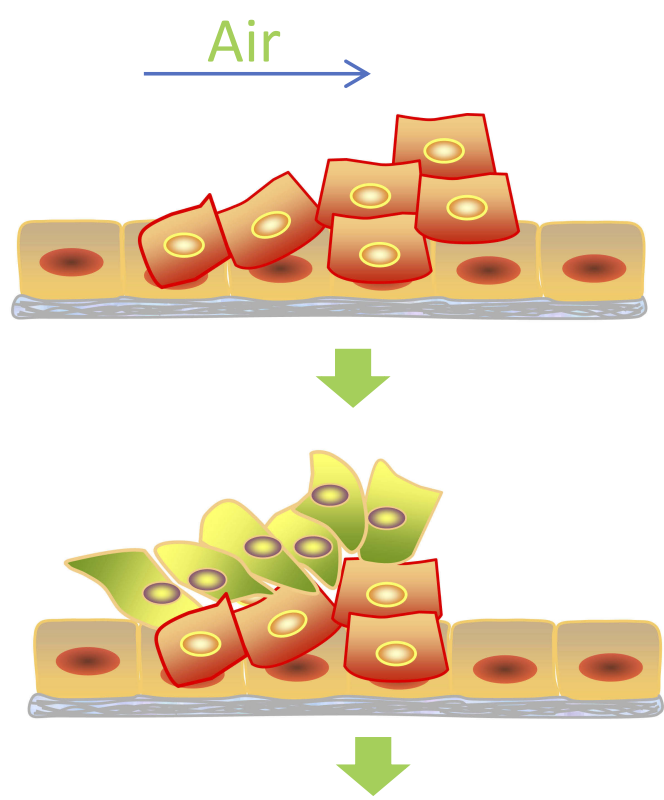

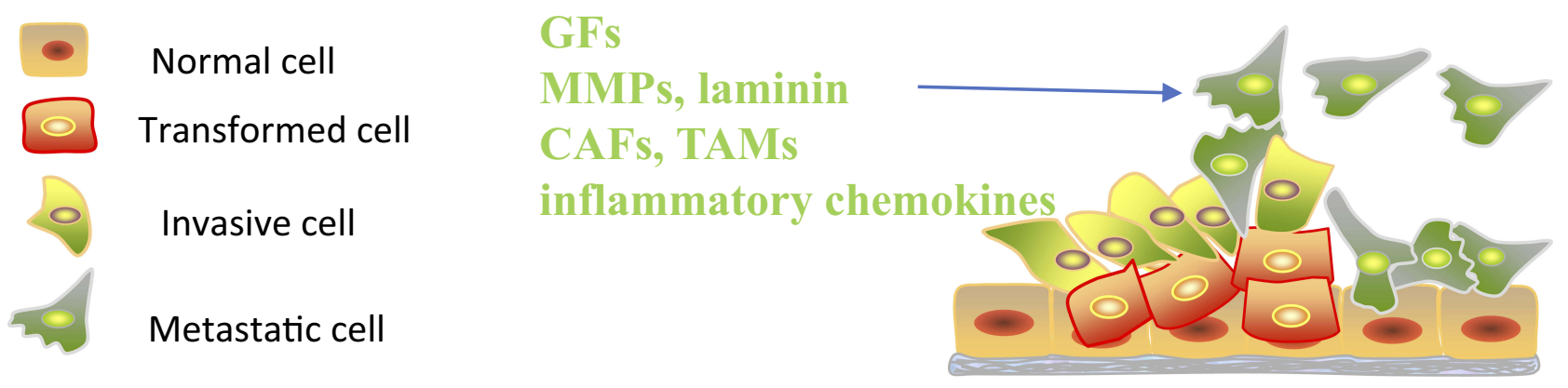

Figure I The potential mechanism of STAS.

Abbreviations: GFs, growth factors; MMPs, matrix metalloproteinases; CAFs, cancer-associated fibroblasts; TAMs, tumor-associated macrophages. 
Warth et al. evaluated 569 resected stage I-IV pulmonary ADCs. They defined a detachment of small solid cell nests (at least 5 tumor cells) $<3$ alveoli away from the main tumor mass as limited STAS and tumor cell nests $>3$ alveoli away from the main tumor mass as extensive STAS. STAS was significantly associated with reduced overall and disease-free survival. ${ }^{5}$ Kadota et al. reviewed 411 resected stage I lung ADCs. STAS was defined as tumor cells including micropapillary structures, solid nests, or single cells that spread within air spaces in the lung parenchyma beyond the edge of the main tumor. They found the presence of STAS correlated with higher risk of distant and locoregional recurrence in the limited resection group, but was not associated with the recurrence in the lobectomy group. ${ }^{10}$

Before the idea of STAS, pathological phenomena similar to tumor STAS were reported. Aerogenous spread was recognized as the presence of isolated clusters of tumor cells in the alveolar space by the 1995 Atlas of Tumor Pathology, but it was underestimated and had not been well studied. Aerogenous spread with floating cancer cell clusters (ASFC) in pulmonary metastases of colorectal carcinoma was first reported in 2005. ${ }^{11}$ The criterion for ASFC was tumor clusters lying free in the alveolar space and at least $0.5 \mathrm{~mm}$ from the main lung metastatic lesion, which is shown to be a prognostic factor for patients who have undergone pulmonary metastasectomy. Onozato et al. ${ }^{12}$ applied a combination of a robotic sectioning system, whole slide imaging technology and 3D-reconstruction software and demonstrated detached, large nests of "island-like" pattern within the alveolar spaces, which were distinct from the micropapillary pattern. Furthermore, the 3D stacking showed connections between these tumor islands and the main tumoral area, which appeared isolated with each other on $2 \mathrm{D}$ view. Then they investigated the clinical and prognostic significance of tumor islands, ${ }^{13}$ and found that lung ADC patients with tumor islands had a significantly worse prognosis than those without tumor islands. They showed that ADCs with tumor islands were more likely to be found in smokers, to show predominant solid or micropapillary growth patterns, and tended to have KRAS mutations. Morimoto et al. ${ }^{14}$ also proposed free tumor clusters (FTCs): a group of $>3$ small clusters containing $<20$ nonintegrated micropapillary tumor cells that were spreading within air spaces, $>3 \mathrm{~mm}$ away from the main tumor. They revealed that coexistence of FTCs made a worse impact on postoperative prognosis among pulmonary adenocarcinoma with a micropapillary component. The result is speculative and needs further investigation.

\section{STAS And The Postoperative Prognosis Of Lung Cancer}

Since the criteria of STAS, many studies have been performed to demonstrate the relationship between STAS and the postoperative prognosis of lung cancer.

In lung cancer, different surgical procedures such as limited resection, wedge resection, and segmentectomy mainly depend on the tumor feature. The relationship between STAS and stage I lung cancer was discussed frequently in the research we reviewed, and investigation ${ }^{15}$ showed that limited resection was associated with a higher risk of recurrence and death than lobectomy in small $(\leq 3 \mathrm{~cm})$ T1N0 lung cancers. Will there be any difference if we introduce the concept of STAS?

\section{STAS In Lung ADC}

Recently, many studies investigated the correlation between STAS and the clinical parameters and prognosis of lung cancer, and the majority of these studies focused on lung ADC.

Kadota et al. ${ }^{10}$ reviewed 411 resected stage I lung ADCs and concluded that the risk of developing locoregional or distant recurrence was significantly higher in patients with STAS than those without STAS in the limited resection group. However, in the lobectomy group, being STAS-positive was not associated with an increased risk of any recurrence, compared with absence of STAS.

A study ${ }^{16}$ was made to compare the effects of STAS and tumor size on recurrence-free survival (RFS) and overall survival (OS). They concluded STAS affects the recurrence and $\mathrm{OS}$ in patients with $\mathrm{ADC}>2-3 \mathrm{~cm}$ and could be considered as a parameter in a staging system to predict prognosis better, especially in ADCs $>2-3 \mathrm{~cm}$. The finding is meaningful and large-scale studies can be done to confirm their findings if possible. Some thought thoracic surgeons should rule out STAS irrespective of the tumor size if limited resection is performed for stage IA lung cancer.

A retrospective study ${ }^{17}$ enrolled 82 patients with earlystage ADCs who underwent limited resection and concluded STAS was predictive of poor postoperative survival in early-stage ADCs treated with limited resection, and was associated with surgical margin recurrence. A study demonstrated that STAS was an independent prognostic 
factor of poor survival in patients with clinical stage IA lung cancer who underwent sublobar resection, but not in the lobectomy group. ${ }^{18}$ Yang et al. ${ }^{19}$ revealed that being STAS-positive was a significantly worse predictor for Stage I patients with lung $\mathrm{ADC}>2 \mathrm{~cm}$ that underwent radical lobectomy, but was not significant in patients with tumor $\leq 2 \mathrm{~cm}$. Eguchi et al. ${ }^{20}$ made a propensity score-matched analysis that including 1497 patients with T1N0M0 lung ADC. They described how pathologists can recognize STAS on frozen sections (FSs), and they made the conclusion that in T1 lung ADC patients with STAS, lobectomy was associated with better outcomes than sublobar resection.

\section{STAS In Lung SCC}

Although most studies were associated with ADCs, the investigators also found that STAS is related to recurrence and survival in patients with lung squamous cell carcinoma (SCC). Lu et al. ${ }^{21}$ reviewed 445 resected stage IIII lung SCCs, and found that the cumulative incidences, distant, and locoregional recurrence as well as lung cancerspecific death were significantly higher in patients with STAS than those without STAS, whereas there was no statistical difference in OS. In multivariable models, STAS was an independent predictor for both recurrence and lung cancer-specific death. A retrospective review ${ }^{22}$ of
220 patients with lung SCC revealed STAS was associated with recurrence and poor survival in stage I SCC, but not in stage II-III SCC. Therefore, they suggested that STAS is a useful predictor of recurrence and prognosis in stage I SCC.

Obviously, there are some differences between STASpositive lung ADC and SCC. All STAS lesions in lung SCC consist of solid tumor cell nests, ${ }^{10}$ while lung ADC with STAS can manifest as micropapillary clusters, solid nests, or single cells. In contrast to lung adenocarcinoma where the presence of STAS was more frequent among more aggressive subtypes, ${ }^{5,10}$ the proportion of STAS was similar in all squamous histologic subtypes. ${ }^{21}$

Liu et al. ${ }^{23}$ evaluated the association between STAS and clinical outcome of lung cancer patients after surgical resection through a meta-analysis with a total of 3564 NSCLC patients. They revealed STAS predicted a worse outcome for 5-year RFS. They also acknowledged lack of SCLC patients to further confirm the conclusion.

The information from some articles associated with early stage non-small cell lung cancer (ADC and SCC) is summarized in Table 1, and we concluded that STAS is a prognostic factor independent of growth pattern and tumor stage, and also an independent risk factor for unfavorable prognosis of stage I lung ADC and stage I lung SCC.

Table I The Studies On Stage I Lung Cancer Corrected With STAS Are Shown As Reported In The Published Studies

\begin{tabular}{|c|c|c|c|}
\hline Study & Entity & $\begin{array}{l}\text { Stage } \\
\text { I (Number) }\end{array}$ & Results \\
\hline Onozato et al. ${ }^{13} 2013$ & ADC & $\begin{array}{l}\text { IA (I5I) } \\
\text { IB (66) }\end{array}$ & $\begin{array}{l}\text { The presence of tumor islands was significantly associated with poor outcomes in the Stage IA } \\
\text { cohort }\end{array}$ \\
\hline Kadota et al. ${ }^{10} 2015$ & ADC & $\mathrm{I}(4 \mid \mathrm{I})$ & The presence of STAS correlated with higher risk of distant and locoregional recurrence \\
\hline Shiono et al. ${ }^{27} 2016$ & ADC & $I(3 \mid 8)$ & STAS was closely related to poor prognosis and recurrence in stage I ADCs \\
\hline Lu et al. ${ }^{21} 2017$ & SCC & I (249) & $\begin{array}{l}\text { In stage I SCC, patients with STAS had higher incidence of locoregional recurrence and lung } \\
\text { cancer-specific death compared to those without STAS }\end{array}$ \\
\hline Uruga et al. ${ }^{42} 2017$ & ADC & I (208) & $\begin{array}{l}\text { One-third of resected small adenocarcinomas had high STAS. Higher STAS was predictive of } \\
\text { worse RFS }\end{array}$ \\
\hline Dai et al ${ }^{16} 2017$ & ADC & $\begin{array}{l}\text { IA (383) } \\
\text { IB (16I) }\end{array}$ & Multivariate analysis revealed STAS to be an independent prognostic factor in stage I ADCs \\
\hline Yanagawa et $\mathrm{al}^{22} 2018$ & SCC & $\mathrm{I}(220)$ & $\begin{array}{l}\text { STAS was associated with recurrence and worse survival in stage I SCC, but not in stage II and } \\
\text { III SCC }\end{array}$ \\
\hline Yang et al ${ }^{19} 2018$ & $A D C$ & I (242) & $\begin{array}{l}\text { STAS was a significantly worse predictor for Stage I patients with lung ADC }>2 \mathrm{~cm} \text { who } \\
\text { underwent radical lobectomy }\end{array}$ \\
\hline Shiono et al. ${ }^{18} 2018$ & NSCLC & IA (5I4) & $\begin{array}{l}\text { STAS is a prognostic factor of poor outcomes for sublobar resection in patients with lung } \\
\text { cancer }\end{array}$ \\
\hline Toyokawa et al. ${ }^{17} 2018$ & $A D C$ & I (276) & $\begin{array}{l}\text { Patients with STAS had significantly shorter RFS and OS than patients without STAS, STAS- } \\
\text { positivity remained an independent prognostic factor for both RFS and OS }\end{array}$ \\
\hline
\end{tabular}

Abbreviations: ADC, adenocarcinoma; SCC, squamous cell carcinoma; STAS, spread through air spaces; NSCLC, nonsmall cell lung carcinoma; RFS, recurrence-free survival; OS, overall survival. 


\section{STAS In Other Histologic Types Of Lung Cancer}

In addition, fewer studies focused on the correlation between STAS and other histologic types of lung cancer. Yokoyama et al. ${ }^{24}$ reviewed tumor specimens of 35 consecutive patients with pleomorphic carcinoma who underwent surgical resection. The results showed that tumor STAS was associated with high recurrence rates and poor survival after surgical resection. Toyokawa et al. ${ }^{25}$ included 30 cases of resected SCLC; however, there was no statistically significant relationship between STAS-positivity and the clinical factors, and no statistical significance was observed about STAS on RFS or OS. We believe their results need to be further confirmed by large-cohort studies.

\section{STAS And Lung Cancer-Related Molecules}

With the development of molecular-targeted therapy and immunotherapy, the detection of molecules such as epidermal growth factor receptor (EGFR), anaplastic lymphoma kinase (ALK), ROS1 and PD-L1 is increasing. The relationship between STAS and lung cancer-related molecules are summarized in Table 2.

Spread was revealed associated with poor diseasefree survival in ROS1-rearranged ADCs. ${ }^{26}$ Warth et al. ${ }^{5}$ found STAS was associated with lower rates of EGFR but higher rates of $B R A F$ mutations, and not associated with KRASmutations, TTF1, napsin or CK7 expression. Shiono et al. ${ }^{27}$ suggested that wild-type EGFR was significantly related to STAS. Toyokawa et al. ${ }^{17}$ concluded that STAS was not significantly associated with $E G F R$ mutations or PD-L1 expression. Lee et al. ${ }^{28}$ concluded that STAS was frequently observed in tumors with wild-type EGFR and ALK rearrangement, and they found no association with KRAS mutation. Although there was no statistical significance with ROS1 rearrangement, when combined with ALK rearrangements, STAS was frequently found in tumors with ALK or ROS1 rearrangements. Kim et al. ${ }^{29}$ concluded that STAS was associated with a lower incidence of EGFR mutation and a higher incidence of ALK rearrangement.

\section{STAS Prediction By Medical Imaging}

STAS is a strong prognostic marker in surgically resected lung cancer reported in many studies. However, STAS could only be detected on surgically resected specimens rather than imaged-guided or bronchoscopic biopsy samples. It could not be identified reliably before the operation, so preoperative prediction of the phenomenon is of great significance. Researchers have made some analysis based on the preoperative computed tomography (CT) and postoperative pathologic features and revealed some interesting results.

Margerie-Mellon et al..$^{30}$ included 80 patients with lung ADCs manifested as subsolid nodules and who underwent surgical resection. The total average diameter of nodules, average and long-axis diameters of the solid component, and the proportion of solid component to overall nodule diameter was significantly larger in the STAS-positive than in the STAS-negative nodules. These findings could serve as an in-vivo tool for the likelihood estimation of STAS, and consequently influence management of subsolid ADCs. Kim et al. ${ }^{29}$ explored CT imaging features for predicting STAS in lung ADCs. They investigated 276 patients who underwent surgical resection and found: (1) STAS was more common in solid tumors than in part-solid or groundglass lesions; (2) STAS was also associated with central low

Table 2 The Relationship Between STAS And Lung Cancer-Related Molecules Are Shown As Reported In The Published Studies

\begin{tabular}{|c|c|c|c|}
\hline Study & Entity & Molecular & Results \\
\hline Warth et al. ${ }^{5} 2015$ & ADC & EGFR BRAF & $\begin{array}{l}\text { STAS was associated with lower rates of EGFR but higher rates of BRAF mutations, and not } \\
\text { associated with KRASmutations }\end{array}$ \\
\hline jin et al. ${ }^{26} 2015$ & ADC & ROSI & STAS was associated with poor DFS in ROSI-rearranged ADCs \\
\hline Shiono et al. ${ }^{27} 2016$ & ADC & EGFR & Wild-type EGFR was significantly related to STAS \\
\hline Toyokawa et al. ${ }^{17} 2018$ & ADC & EGFR PD-LI & STAS was not significantly associated with EGFRmutations or PD-LI expression \\
\hline Lee et al. ${ }^{28} 2018$ & ADC & EGFR ALK & $\begin{array}{l}\text { STAS was frequently observed in tumors with wild-type EGFR and ALK rearrangement, and } \\
\text { they found no association with KRASmutation }\end{array}$ \\
\hline Kim et al. ${ }^{29} 2018$ & ADC & EGFR ALK & $\begin{array}{l}\text { STAS was associated with a lower incidence of EGFR mutation }(P=0.02) \text { and a higher } \\
\text { incidence of ALK rearrangement }(P=0.003)\end{array}$ \\
\hline
\end{tabular}

Abbreviations: ADC, adenocarcinoma; EGFR, epidermal growth factor receptor; ROSI, c-ros oncogene I; ALK, anaplastic lymphoma kinase; STAS, spread through air spaces; DFS, disease-free survival. 
attenuation, ill-defined opacity, air bronchogram, and percentage of solid component (PSC); and (3) PSC was an independent predictor of STAS and a cut-off value of $90 \%$ showed a discriminatory power with a sensitivity of $89.2 \%$ and a specificity of $60.3 \%$. Toyokawa et al. ${ }^{31}$ investigated 327 patients with resected ADCs, and in a multivariable analysis, the presence of notch and the absence of groundglass opacity were shown to be significantly associated with the STAS phenomenon. The magnitude of STAS is so small that this phenomenon may not reliably be detected on CT, as many other factors may contribute to a change in CT. We still hope the above results will be helpful in identifying STAS-positive ADC by $\mathrm{CT}$ before surgical resection someday.

\section{STAS Exists Or Not}

STAS has been proved with high correlation to prognosis and recurrence of lung cancer, but the concept of STAS is still controversial. Is STAS an in-vivo effect or potentially an ex-vivo artifact? Some researchers have made different opinions. Surgical and pathologic processing of lung physical effects on lung tissue are sometimes inevitable. Tumor contamination of a needle biopsy with subsequent recurrence of the tumor along that biopsy path has been reported in lung cancer many times, ${ }^{32-35}$ and if the tumor was then resected, pathologists might find STAS. Thunnissen et al. ${ }^{36}$ illustrated 4 patterns of artifacts, one of which is tissue fragments and individual cells spreading through a knife surface (STAKS). They used an artificial model of an apple with ink to show displaced ink in the direction of cutting. Blaauwgeers et al. ${ }^{37}$ suggested that overall $93 \%$ of the loose tissue fragments could be explained by mechanical forces associated with tissue handling. In many instances, STAS likely represents mechanical artifacts including knife spread during specimen section. They thought it might be premature to recognize STAS as a morphologic pattern of lung cancer invasion.

Undoubtedly, the artifacts during surgeon and pathological specimen procession exist. However, the 3D reconstruction showed connections between these tumor islands and the main tumoral area. $\mathrm{Lu}$ et al. ${ }^{38}$ presented three cases which provide evidence that STAS was not an artifact, among which in two cases the extensive STAS predominant pattern was not a knife-cutting artifact because the main tumor was not cut either by the surgeon or the pathologist. STAS was the only pattern of tumor identified at a wedge resection margin in another case, and the residual and metastatic tumor would not have been identified, delaying introduction of chemotherapy if STAS was ignored.

\section{Difficulties Associated With STAS Pathologically}

There may be limitations in the search for STAS in tumors arising within a fibrotic underlying lung parenchyma and in cases where the submitted histologic sections did not sample the edge of the tumor including the surrounding non-neoplastic lung. ${ }^{21}$

STAS should be distinguished from alveolar macrophages by morphologic features ${ }^{10,21}$ where STAS showed a high N/C ratio and nuclear atypia, while macrophages showed small nuclei and cytoplasm and sometimes contained carbon pigment, while in nonsmokers the pigment is lack and the cytoplasm is sometimes foamy. Tumor budding, which is defined as the presence of isolated single cancer cells or a cluster of cancer cells composed of fewer than 5 cells in the stroma at the outer edge of the tumor, was sometimes difficult to distinguish from STAS. However, tumor budding was observed within the tumor stroma, while STAS was observed within air spaces in the alveolar parenchyma. Immunohistochemistry for keratin and a macrophage marker such as CD68 may be helpful.

STAS should also be distinguished from artifacts. To avoid confusion with artificially detached or floating cells during tumor dissection, tumor cells were considered as STAS only if they appeared as detached small clusters or if they were arranged in loose small groups within air spaces in a continuous manner from the edge of the tumor, and the distribution was consistent with the overall configuration of the circumferential tumor edge. ${ }^{5,21}$ Haphazardly distributed fragments of tumor with sharp jagged edges were regarded as artifacts. ${ }^{21}$

\section{Conclusions And Perspectives}

Most research involving STAS has demonstrated that this novel morphologic feature is of significant prognostic value. The results indicated that STAS is associated with pivotal clinical variables and the prognosis of patients in lung ADC, lung SCC, small cell lung cancer, and lung pleomorphic carcinoma. We conclude that STAS is a prognostic factor independent of growth pattern and tumor stage, and is also an independent risk factor for unfavorable prognosis of stage I lung ADC and stage I lung SCC. 
STAS is not visible to pathologists on gross exam, and is easily ignored because most pathologists are not trained to look for STAS routinely. Surgical specimens which include the circumferential tumor edge as well as adjacent lung parenchyma are very necessary for seeking STAS. Up to now, we have no pathologic standards.

Intraoperative frozen section is possible to detect STAS; however, some researchers ${ }^{39-41}$ have demonstrated that the identification showed high specificity but very low sensitivity. Eguchi et al. ${ }^{20}$ made a propensity score-matched analysis, which showed the sensitivity and specificity for detecting STAS by use of FSs were $71 \%$ and $92 \%$; they suggested that pathologists can recognize STAS on FSs. We really need pathological standards for STAS on intraoperative FSs.

We think STAS is an independent risk factor for recurrence, independent of type of surgery. If STAS is detected by FSs, limited resection should be avoided, as this will lead to higher risk of recurrence in patients. If STAS is detected postoperatively, treatment after operation needs to be discussion seriously. However, if STAS is misdetected intraoperatively, an extended operation may do harm to the patient. It's significant to distinguish STAS from artifact and to make pathological standards for STAS on intraoperative FSs.

A study ${ }^{16}$ showed that patients with solid and micropapillary-predominant ADCs received the most benefits from adjuvant chemotherapy. Given that patients with solid and micropapillary-predominant ADCs reportedly have a high prevalence of STAS, $5,10,42$ adjuvant chemotherapy might be needed in STAS-positive patients after surgery.

In conclusion, STAS is nowadays seen more and more in lung cancer. It is associated with multiple pathologic and clinical features in both ADC and SCC. It's also an important prognostic feature independent of tumor stage and ADC growth patterns. The mechanism is still not well studied. It should be paid more attention and recorded in pathological reports, which can introduce treatment choice and indicate prognosis of lung cancer patients. A more precise standard of pathology is needed to avoid superfluous harm to patients in the meantime.

Although there are lot of studies to suggest the existence of STAS, the debate whether STAS is an in-vivo effect or an artifact is fierce. We need to collect as much evidence as possible to make it more reasonable.

In future, more well-designed studies with large cohorts are needed to confirm the clinicopathological and prognostic studies on STAS.

\section{Disclosure}

The authors declare no conflicts of interest in this work.

\section{References}

1. Chen W, Zheng R, Baade PD, et al. Cancer statistics in China, 2015. CA Cancer J Clin. 2016;66(2):115-132. doi:10.3322/caac.21338

2. Siegel RL, Miller KD, Jemal A. Cancer statistics, 2019. CA Cancer J Clin. 2019;69(1):7-34. doi:10.3322/caac.v69.1

3. Travis WD, Brambilla E, Nicholson AG, et al. The 2015 world health organization classification of lung tumors. $J$ Thorac Oncol. 2015;10 (9):1243-1260. doi:10.1097/JTO.0000000000000630

4. Shiono S, Endo M, Suzuki K, et al. Spread through air paces in lung cancer patients is a risk factor for pulmonary metastasis after surgery. J Thorac Dis. 2019;11(1):177-187. doi:10.21037/jtd.2018.12.21

5. Warth A, Muley T, Kossakowski CA, et al. Prognostic impact of intra-alveolar tumor spread in pulmonary adenocarcinoma. Am J Surg Pathol. 2015;39(6):793-801. doi:10.1097/PAS.0000000000000409

6. Liotta LA. Adhere, degrade, and move: the three-step model of invasion. Cancer Res. 2016;76(11):3115-3117. doi:10.1158/00085472.CAN-16-1297

7. Lijing C, Gang C, Dongchang W. Airway disseminated intrapulmonary metastasis in lung adenocarcinoma. Int $J$ Respir. 2016;36(11): 871-874

8. Wislez M, Antoine M, Rabbe N, et al. Neutrophils promote aerogenous spread of lung adenocarcinoma with bronchioloalveolar carcinoma features. Clin Cancer Res. 2007;13(12):3518-3527. doi:10.11 58/1078-0432.CCR-06-2558

9. Qiu X, Chen D, Liu Y, et al. Relationship between stromal cells and tumor spread through air spaces in lung adenocarcinoma. Thorac Cancer. 2019;10:256-267. doi:10.1111/tca.2019.10.issue-2

10. Kadota K, Nitadori J, Sima CS, et al. Tumor spread through air spaces is an important pattern of invasion and impacts the frequency and location of recurrences after limited resection for small Stage I lung adenocarcinomas. J Thorac Oncol. 2015;10(5):806-814.

11. Shiono S, Ishii G, Nagai K, et al. Predictive factors for local recurrence of resected colorectal lung metastases. Ann Thorac Surg. 2005;80(3):1040-1045. doi:10.1016/j.athoracsur.2004.12.033

12. Onozato ML, Klepeis VE, Yagi Y, et al. A role of three-dimensional (3D)-reconstruction in the classification of lung adenocarcinoma. Anal Cell Pathol (Amst). 2012;35(2):79-84. doi:10.1155/2012/684 751

13. Onozato ML, Kovach AE, Yeap BY, et al. Tumor islands in resected early-stage lung adenocarcinomas are associated with unique clinicopathologic and molecular characteristics and worse prognosis. Am J Surg Pathol. 2013;37(2):287-294. doi:10.1097/PAS.0b013e31826885fb

14. Morimoto J, Nakajima T, Suzuki H, et al. Impact of free tumor clusters on prognosis after resection of pulmonary adenocarcinoma. $J$ Thorac Cardiovasc Surg. 2016;152(1):64-72. doi:10.1016/j.jtcvs.2016.03.088

15. Ginsberg RJ, Rubinstein LV. Randomized trial of lobectomy versus limited resection for T1 N0 non-small cell lung cancer. Lung Cancer Study Group. Ann Thorac Surg. 1995;60(3):615-622, 622-623. doi:10.1016/0003-4975(95)00537-U

16. Dai C, Xie H, Su H, et al. Tumor spread through air spaces affects the recurrence and overall survival in patients with lung adenocarcinoma $>2$ to $3 \mathrm{~cm}$. $J$ Thorac Oncol. 2017;12(7):1052-1060. doi:10.1016/j. jtho.2017.03.020

17. Toyokawa G, Yamada Y, Tagawa T, et al. Significance of spread through air spaces in resected pathological Stage I lung adenocarcinoma. Ann Thorac Surg. 2018;105(6):1655-1663. doi:10. 1016/j.athoracsur.2018.01.037

18. Shiono S, Endo M, Suzuki K, et al. Spread through air spaces is a prognostic factor in sublobar resection of non-small cell lung cancer. Ann Thorac Surg. 2018;106(2):354-360. doi:10.1016/j. athoracsur.2018.02.076 
19. Yang L, Yang Y, Ma P, et al. Spread through air spaces predicts a worse survival in patients with stage I adenocarcinomas $>2 \mathrm{~cm}$ after radical lobectomy. $J$ Thorac Dis. 2018;10(9):5308-5317. doi:10.21 037/jtd.2018.09.22

20. Eguchi T, Kameda K, Lu S, et al. Lobectomy is associated with better outcomes than sublobar resection in Spread through Air Spaces (STAS)-positive T1 lung adenocarcinoma: a propensity score-matched analysis. J Thorac Oncol. 2019;14(1):87-98. doi:10.1016/j.jtho.2018. 09.005

21. Lu S, Tan KS, Kadota K, et al. Spread through air spaces (STAS) is an independent predictor of recurrence and lung cancer-specific death in squamous cell carcinoma. J Thorac Oncol. 2017;12(2):223-234. doi:10.1016/j.jtho.2016.09.129

22. Yanagawa N, Shiono S, Endo M, et al. Tumor spread through air spaces is a useful predictor of recurrence and prognosis in stage I lung squamous cell carcinoma, but not in stage II and III. Lung Cancer. 2018;120:14-21. doi:10.1016/j.lungcan.2018.03.018

23. Liu H, Yin Q, Yang G, et al. prognostic impact of tumor spread through air spaces in non-small cell lung cancers: a meta-analysis including 3564 patients. Pathol Oncol Res. 2019;25(4):1303-1310.

24. Yokoyama S, Murakami T, Tao H, et al. Tumor spread through air spaces identifies a distinct subgroup with poor prognosis in surgically resected lung pleomorphic carcinoma. Chest. 2018;154(4):838-847. doi:10.1016/j.chest.2018.06.007

25. Toyokawa G, Yamada Y, Tagawa T, et al. High frequency of spread through air spaces in resected small cell lung cancer. Anticancer Res. 2018;38(3):1821-1825. doi:10.21873/anticanres.12421

26. Jin Y, Sun PL, Park SY, et al. Frequent aerogenous spread with decreased E-cadherin expression of ROS1-rearranged lung cancer predicts poor disease-free survival. Lung Cancer. 2015;89 (3):343-349. doi:10.1016/j.lungcan.2015.06.012

27. Shiono S, Yanagawa N. Spread through air spaces is a predictive factor of recurrence and a prognostic factor in stage I lung adenocarcinoma. Interact Cardiovasc Thorac Surg. 2016;23(4):567-572. doi:10.1093/ icvts/ivw211

28. Lee JS, Kim EK, Kim M, Shim HS. Genetic and clinicopathologic characteristics of lung adenocarcinoma with tumor spread through air spaces. Lung Cancer. 2018;123:121-126. doi:10.1016/j.lungcan.20 18.07 .020

29. Kim SK, Kim TJ, Chung MJ, et al. Lung adenocarcinoma: CT features associated with spread through air spaces. Radiology. 2018;289(3):831-840. doi:10.1148/radiol.2018180431

30. de Margerie-mellon C, VanderLaan PA, Bankier AA, et al. CT manifestations of tumor spread through air spaces in lung adenocarcinoma: different pathways toward common perspectives. Radiology. 2019;290(1):271-272. doi:10.1148/radiol.2018182173
31. Toyokawa G, Yamada Y, Tagawa T, et al. Computed tomography features of resected lung adenocarcinomas with spread through air spaces. J Thorac Cardiovasc Surg. 2018;156(4):1670-1676. doi:10. 1016/j.jtcvs.2018.04.126

32. Valle LG, Rocha RD, Mendes GF, et al. Tumor seeding along the needle track after percutaneous lung biopsy. J Bras Pneumol. 2016;42(1):71. doi:10.1590/S1806-37562016000000280

33. Scotti V, Di Cataldo V, Falchini M, et al. Isolated chest wall implantation of non-small cell lung cancer after fine-needle aspiration: a case report and review of the literature. Tumori. 2012;98(5):126e129e. doi: $10.1177 / 030089161209800522$

34. Matsuguma H, Nakahara R, Kondo T, et al. Risk of pleural recurrence after needle biopsy in patients with resected early stage lung cancer. Ann Thorac Surg. 2005;80(6):2026-2031. doi:10.1016/j. athoracsur.2005.06.074

35. McDonald CF, Baird L. Risk of needle track metastasis after fine needle lung aspiration in lung cancer-a case report. Respir Med. 1994;88(8):631-632. doi:10.1016/S0954-6111(05)80013-7

36. Thunnissen E, Blaauwgeers HJ, de Cuba EM, et al. Ex vivo artifacts and histopathologic pitfalls in the lung. Arch Pathol Lab Med. 2016;140(3):212-220. doi:10.5858/arpa.2015-0292-OA

37. Blaauwgeers H, Flieder D, Warth A, et al. A prospective study of loose tissue fragments in non-small cell lung cancer resection specimens: an alternative view to "spread through air spaces". Am J Surg Pathol. 2017;41(9):1226-1230. doi:10.1097/PAS.0000000000000889

38. Lu S, Rekhtman N, Eguchi T, et al. Cases demonstrating Spread Through Air Spaces (STAS) reflects invasive growth and not an artifact. J Thorac Oncol. 2016;12(1):S1137. doi:10.1016/j.jtho.2016. 11.1595

39. Walts AE, Marchevsky AM. Current evidence does not warrant frozen section evaluation for the presence of tumor spread through alveolar spaces. Arch Pathol Lab Med. 2018;142(1):59-63. doi:10. 5858/arpa.2016-0635-OA

40. Trejo BH, Incharoen P, Althouse AD, et al. Accuracy of the IASLC/ ATS/ERS histological subtyping of stage I lung adenocarcinoma on intraoperative frozen sections. Mod Pathol. 2015;28(8):1058-1063. doi:10.1038/modpathol.2015.71

41. Yoshida J, Nagai K, Yokose $\mathrm{T}$, et al. Limited resection trial for pulmonary ground-glass opacity nodules: fifty-case experience. J Thorac Cardiovasc Surg. 2005;129(5):991-996. doi:10.1016/j.jtcvs. 2004.07.038

42. Uruga H, Fujii T, Fujimori S, et al. Semiquantitative assessment of tumor spread through air spaces (STAS) in early-stage lung adenocarcinomas. $J$ Thorac Oncol. 2017;12(7):1046-1051. doi:10.10 16/j.jtho.2017.03.019

\section{Publish your work in this journal}

Cancer Management and Research is an international, peer-reviewed open access journal focusing on cancer research and the optimal use of preventative and integrated treatment interventions to achieve improved outcomes, enhanced survival and quality of life for the cancer patient.
The manuscript management system is completely online and includes a very quick and fair peer-review system, which is all easy to use. Visit http://www.dovepress.com/testimonials.php to read real quotes from published authors. 